\title{
Mesures de profils de densité d'espèces par radiographie rapide sur des jets gazeux et liquides
}

\author{
L. Huré, E. Robert, C. Cachoncinlle, R. Viladrosa et J.-M. Pouvesle
}

GREMI, EPEE FR 0776, BP. 6744, 45067 Orléans cedex 2, France

\begin{abstract}
Résumé : Avec le développement des scanners médicaux au début des années 1970, sont apparues les premières techniques de tomographie par transmission qui font appel à des algorithmes de reconstruction d'image. Dans ces travaux, nous présentons un algorithme de calcul simple, qui permet de mesurer des densités d'espèces sur des milieux radiographiés par flash X. Des profils de densité d'espèces ont ainsi été obtenus sur des milieux peu denses tels que des écoulements gazeux et liquides. L'algorithme de calcul a été validé sur des jets d'argon et d'eau. Ce diagnostic est un outil complémentaire pour caractériser les écoulements en sortie des systèmes d'injection.
\end{abstract}

\section{PRINCIPE EXPERIMENTAL}

La source flash $\mathrm{X}$ utilisée, développée au GREMI, est une source compacte qui émet des impulsions de photons $\mathrm{X}$ sur de très courtes durées $(20 \mathrm{~ns}$ ). Ces photons proviennent essentiellement des émissions des raies caractéristiques du matériau constitutif de l'anode, fer ou tungstène dans les travaux présentés. Leur absorption lors de la traversée de milieux peu denses tels que écoulements gazeux ou liquides est suffisante pour révéler des contrastes significatifs sur des clichés radiographiques. Cette technique de diagnostic par radiographie rapide a été validée sur des jets d'argon se détendant dans l'air ambiant à pression atmosphérique [1]. Ces jets se détendent parallèlement, à quelques centimètres du film photographique à travers des buses cylindriques situées à des distances comprises entre 10 et $30 \mathrm{~cm}$ de la source flash X. Les films sont développés puis numérisés sur 256 niveaux de gris. L'algorithme de calcul développé, basé sur un traitement numérique des images radiographiques, permet de mesurer les densités d'espèces présentes dans les jets.

\section{DESCRIPTION DE L'ALGORITHME}

Dans l'algorithme, le foyer de la source flash $\mathrm{X}$ dont la taille réelle n'excède pas $300 \mu \mathrm{m}$, est considéré comme ponctuel. A proximité des buses, la géométrie de l'écoulement peut être estimée comme étant cylindrique. Dans ces conditions, une coupe radiale du jet forme un disque qui peut être entièrement décrit par la mesure du profil de densité d'espèces selon son rayon. Ce disque est découpé en un ensemble de couronnes concentriques d'isodensité, comme le montre la figure 1.

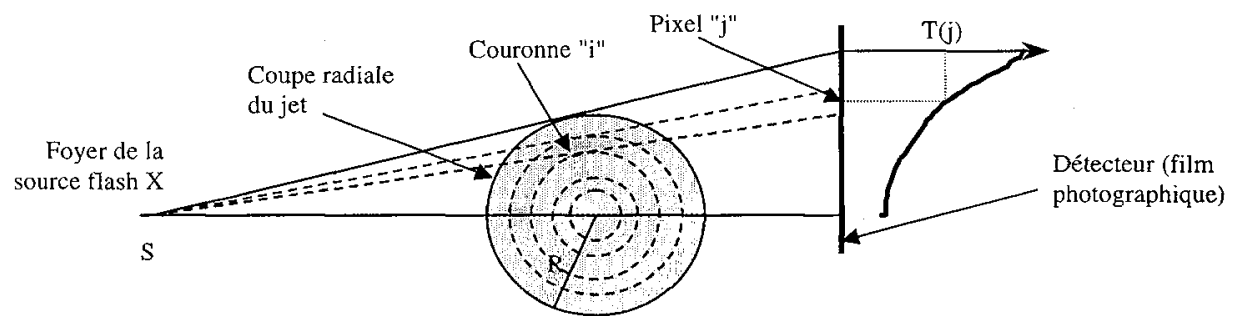

Figure 1: Schéma de principe de la détermination des profils radiaux de densité d'espèces.

La transmission $T(j)$ d'un faisceau de rayons $X$ couvrant le pixel noté " $\mathrm{j}$ " de l'image radiographique dépend de l'épaisseur traversée dans chaque couronne " $i$ " de densité d'espèces $\rho_{i}$ suivant la relation de Beer - Lambert (1). 


$$
\mathrm{T}(\mathrm{j})=\exp \left(-\alpha_{\mathrm{E}} \sum_{i}^{j} \rho_{\mathrm{i}} \times e_{i, j}\right)
$$

$e_{i, j}$ est l'épaisseur traversée dans la couronne de densité $\rho_{i}$ par le faisceau de rayons $X$ couvrant le pixel noté " $j " . \alpha_{E}$ représente le coefficient d'atténuation massique et $s^{\prime}$ exprime $\mathrm{en}^{2} \cdot \mathrm{gm}^{-1}$. Il est une caractéristique du milieu traversé et dépend de l'énergie du rayonnement $\mathrm{X}$ incident. Dans le cadre de ce travail, pour simplifier l'algorithme de calcul, on considère la source comme étant monochromatique. Ainsi, pour une anode en tungstène, une énergie de $9 \mathrm{keV}$ est attribuée aux photons $\mathrm{X}$ incidents (respectivement $7 \mathrm{keV}$ pour une anode en fer). Le calcul des densités d'espèces sur les couronnes successives se déduit par inversion de la relation (1).

\section{RESULTATS}

Sur la figure 2 sont présentés la radiographie d'un jet d'argon (-a-), son profil de transmission selon la coupe $\mathrm{AA}^{\prime}(-\mathrm{b}-)$ et le profil radial de densité d'argon calculé (-c-). Sur cet exemple, le rayonnement $\mathrm{X}$ provient d'une anode en tungstène. A une pression d'un bar d'argon correspond une densité volumique de $1,610^{-3} \mathrm{~g} \cdot \mathrm{cm}^{-3}$. Cette valeur est retrouvée par le calcul au centre du jet.
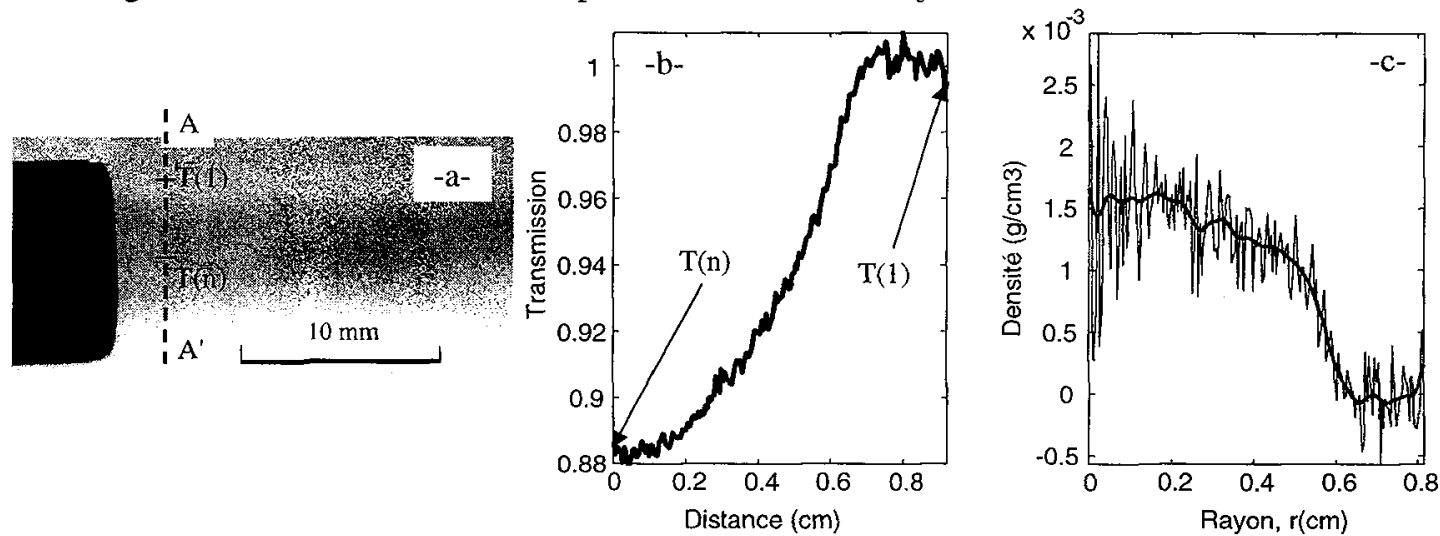

Figure 2: -a- Radiographie rapide d'un jet d'argon se détendant dans l'air ambiant, à pression atmosphérique, soumis à un rayonnement $X$ provenant d'une anode en tungstène. -b- Profil de transmission sur le cliché radiographique selon la coupe $\mathrm{AA}^{\prime}$ entre le centre $T(n)$ et l'extérieur $T(1)$ du jet. -c- Densité volumique d'argon calculée selon le rayon du jet.

Des mesures de densités d'argon identiques sont obtenus en remplaçant l'anode en tungstène par une anode en fer. Elles fournissent des résultats similaires pour un rayonnement $\mathrm{X}$ incident de $7 \mathrm{keV}$. D'autres mesures ont été effectuées sur des jets composés d'un mélange argon (70\%)- azote (30\%) se détendant à un bar. Dans ces conditions l'azote est quasi transparent au rayonnement $\mathrm{X}$ incident et la densité d'argon au centre du jet, calculée par l'algorithme correspond à la valeur théorique $\left(10^{-3} \mathrm{~g} \cdot \mathrm{cm}^{-3}\right)$. Des études ont également été menées sur des filets d'eau de quelques millimètres d'épaisseur, se détendant dans l'air ambiant. L'algorithme de calcul redonne là aussi, la densité volumique de l'eau. Ces expériences sur des milieux de diverses natures ont servi à tester puis à valider l'algorithme de calcul.

Des mesures locales de densité d'espèces dans des jets gazeux et liquides peuvent ainsi être ajoutées aux radiographies rapides déjà obtenues [1]. Ces études sont particulièrement intéressantes pour améliorer les techniques de diagnostics sur des écoulements peu denses et en particulier pour les dispositifs d'injection.

\section{Remerciements}

Ce travail bénéficie du soutien financier du MENRT et de la fédération de recherche EPEE (FR 0776)

\section{Référence}

[1] J. Geiswiller et al, Meas. Sci. and Technol. 2 (1998) 1537-1542 